\title{
THE PROBLEM OF BOLZA IN THE CALCULUS OF VARIATIONS
}

\author{
M. R. HESTENES
}

1. Introduction. The problem of Bolza can be described briefly as the most general problem in the calculus of variations for which there exists at the present time a theory of relative maxima and minima that is comparable in completeness to those of the simpler problems in the calculus of variations. This completeness has been brought about in the last few years and it is the purpose of the present paper to discuss in some detail the results that have been achieved and the methods that have been used in obtaining them. It is impossible to give here an adequate discussion of the various aspects of the problem of Bolza. Nor is such a discussion necessary, inasmuch as three excellent reports have been given already which were concerned in whole or in part with the problem of Bolza. The first of these reports, given in 1936 by Professor Bliss [14], ${ }^{1}$ is devoted to the study of the evolution of problems in the calculus of variations and, in particular, the evolution of the problem of Bolza. Professor Bliss pointed out that even Euler and Lagrange formulated problems that are of essentially the same generality as the problem of Bolza. Moreover, they derived in a formal way the Euler-Lagrange equations that the solutions of the problems must satisfy. The second report was given in 1937 by Professor Reid [17] and was concerned with boundary value problems and their relations to problems in the calculus of variations. In particular, he pointed out the various relationships between the problem of Bolza and boundary value problems. The third and final report was given in 1938 by Professor McShane [22] who outlined the progress that had been made in the calculus of variations during the preceding twenty-five years. Here the essential achievements in the theory of the problem of Bolza were described but nothing was said as to how these results were obtained. It is the purpose of the present paper to discuss certain interesting and important aspects of the theory of the problem of Bolza that Professors Bliss, Reid and McShane of necessity had to omit or describe inadequately in their reports. In particular I shall describe to you the basic ideas involved in the sufficiency proofs for relative minima. The progress made in

An address delivered before the Chicago meeting of the Society on April 11, 1941, by invitation of the Program Committee; received by the editors June 2, 1941.

${ }^{1}$ Numbers in brackets refer to the list of papers at the end of this paper. 
the technique of sufficiency proofs not only has enabled us to obtain better sufficiency theorems but also has in a sense unified the sufficiency proofs of the fixed end point problems and the variable end point problems.

There are certain phases of the problem of Bolza that I too must omit in the present report. I shall not discuss the parametric case; nor shall I have the occasion to describe the Hamilton Jacobi theory and the interesting approach to the theory of the problem of Lagrange made by Carathéodory. I shall not describe how the theory can be modified so as to take care of discontinuous solutions and many problems that are essentially of Bolza type. Finally I shall omit the important topic of existence theorems for the problems of Bolza and Mayer developed by Graves, McShane and others. References to these and other topics can be found in the papers listed at the end of the present paper.

2. Formulation of the problem. The problem of Bolza can be formulated in a number of ways, each of which has its peculiar advantages and disadvantages. One of the most useful formulations is the one given by Professor Bliss [4] in 1932. This formulation closely resembles the original one given by Bolza [1] in 1913 and can be described briefly as follows: Consider a class of arcs

$$
y_{i}(x), \quad x_{1} \leqq x \leqq x_{2} ; i=1, \cdots, n,
$$

in $\left(x y_{1} \cdots y_{n}\right)$-space satisfying a prescribed set of differential equations and end conditions

$$
\begin{aligned}
\phi_{\gamma}\left(x, y, y^{\prime}\right) & =0, & \gamma=1, \cdots, m<n, \\
\psi_{\mu}\left[x_{1}, y\left(x_{1}\right), x_{2}, y\left(x_{2}\right)\right] & =0, & \mu=1, \cdots, p \leqq 2 n+2 .
\end{aligned}
$$

We seek to minimize in this class a function of the form

$$
I=g\left[x_{1}, y\left(x_{1}\right), x_{2}, y\left(x_{2}\right)\right]+\int_{x_{1}}^{x_{2}} f\left(x, y, y^{\prime}\right) d x .
$$

The case when $g=0$ is the so-called problem of Lagrange and when $f=0$ we have the general problem of Mayer as formulated by Bliss. These three problems are equivalent in the sense that each can be transformed into either one of the other two types. Of the three problems, the problem of Bolza appears to be the most convenient.

The formulation of the problem of Bolza just described has been one of the most popular ones in recent years and for many purposes it is the most convenient one. However, there is a second formulation that appears to be more useful for the purposes of the present report. 
It is a modification of one given by Professor Morse [3] and can be described as follows: Consider a class of arcs

$$
a_{h}, y_{i}(x), \quad x_{1} \leqq x \leqq x_{2} ; h=1, \cdots, r ; i=1, \cdots, n,
$$

in $a x y$-space whose components $a_{h}$ are independent of $x$ and which satisfy conditions of the form

$$
\begin{aligned}
\phi_{\gamma}\left(a, x, y, y^{\prime}\right) & =0, & \gamma=1, \cdots, m<n, \\
x_{s}=x_{s}(a), & y_{i}\left(x_{s}\right)=y_{i s}(a), & s=1,2 ; i=1, \cdots, n .
\end{aligned}
$$

We seek to minimize in this class a function of the form

$$
I=g(a)+\int_{x_{1}}^{x_{2}} f\left(a, x, y, y^{\prime}\right) d x .
$$

This formulation differs from the one first described in that certain components of our arcs, denoted by $a_{h}$, are known to be constants. If we consider these constants as functions $a_{h}(x)$ satisfying the differential equations $a_{h}^{\prime}(x)=0$ and if the $a$ 's appearing in the functions $x_{s}(a), y_{i s}(a), g(a)$ are replaced by $a_{h}\left(x_{1}\right)$, the problem becomes one of the type formulated by Bliss. On the other hand, the first formulation can be transformed into one of the second type by a simple device.

I remarked a moment ago that the formulation of the problem of Bolza just described is a modification of one given by Morse. It differs from the one given by Professor Morse in that we have introduced the parameters $a_{h}$ in the functions $f$ and $\phi_{\gamma}$. Although no generality is gained by this change, it has been made for the following two reasons: In the first place in the study of Mayer fields one is led in a natural way to the study of an auxiliary problem in which the parameters $a_{h}$ appear in the integrand. Thus by introducing these parameters in the original integrand one is able to apply at once to the auxiliary problem the results previously obtained. This procedure is analogous to the one introduced by Bliss in the study of the second variation and simplifies the arguments that have to be made. A second trivial advantage is that the class of problems that are immediate special cases is enlarged, although the class of problems that can be transformed into one of Bolza type is unaltered.

No discussion of the problem of Bolza would be complete if it did not include a consideration of the isoperimetric problem of Bolza [24, 25 ]. This problem differs from the one just described in that the arcs (5) are required to satisfy, besides equations (6) and (7), a set of isoperimetric conditions 


$$
I_{\rho}=g_{\rho}(a)+\int_{x_{1}}^{x_{2}} f_{\rho}\left(a, x, y, y^{\prime}\right) d x=0, \quad \rho=1, \cdots, p .
$$

Although this problem can be reduced to one of Bolza type, the present theory for the problem of Bolza does not give adequate sufficiency theorems for this problem. An additional argument is needed. I shall discuss this point later. I would like to point out, however, that once one has obtained a satisfactory theory for the isoperimetric problem of Bolza, these results can be applied at once to a large variety of problems. In particular, they can be applied to the usual isoperimetric problems and to the more complicated problem of minimizing functions of integrals. The latter problem was proposed by Euler and has been studied recently by Brady [23].

3. Normality. One of the most important advances in the theory of the problem of Bolza has been in the elimination of undesirable assumptions of normality. The concept of normality arises when one considers the problem of imbedding a particular arc $E$ satisfying equations (6) and (7) in a one-parameter family of arcs

$$
a_{h}(t), \quad y_{i}(x, t), \quad x_{1}(t) \leqq x \leqq x_{2}(t),
$$

satisfying these equations. If the family (10) contains $E$ for $t=0$, its variations

$$
\alpha_{h}=a_{h}^{\prime}(0), \quad \eta_{i}(x)=y_{i t}(x, 0), \quad x_{1} \leqq x \leqq x_{2},
$$

along $E$ are solutions of equations of the form

$$
\begin{aligned}
\Phi_{\gamma}\left(\alpha, x, \eta, \eta^{\prime}\right) & =\phi_{\gamma a_{h}} \alpha_{h}+\phi_{\gamma y_{i} \eta_{i}}+\phi_{\gamma y_{i}} \eta_{i}^{\prime}=0, \gamma=1, \cdots, m, \\
\eta_{i}\left(x_{s}\right) & =c_{i s h} \alpha_{h}, \quad s=1,2 ; i=1, \cdots, n .
\end{aligned}
$$

Unfortunately, not all solutions $\alpha_{h}, \eta_{i}$ of these equations are necessarily variations (11) of a family (10) that satisfies equations (6) and (7) and contains $E$ for $t=0$. Perhaps the simplest criterion that will insure this property is the existence of a set of $2 n$ solutions $\alpha_{h j}, \eta_{i j}(x)$ $(j=1, \cdots, 2 n)$ of equations (12) whose determinant

$$
\left|\begin{array}{c}
\eta_{i j}\left(x_{1}\right)-c_{i 1 h} \alpha_{h j} \\
\eta_{i j}\left(x_{2}\right)-c_{i 2 h} \alpha_{h j}
\end{array}\right|, \quad j=1, \cdots, 2 n,
$$

is different from zero. If this criterion is satisfied, the arc $E$ is said to be normal relative to the end conditions (7). It is clear that $E$ will be normal if the determinant (14) can be made different from zero by a set of $2 n$ solutions $\alpha_{h j}, \eta_{i j}(x)$ of equations (12) having $\alpha_{h j}=0$. If this stronger condition is satisfied, the arc $E$ is said to be normal on 
the interval $x_{1} x_{2}$, inasmuch as this criterion is a condition of normality for the fixed end point problem obtained by holding the $a$ 's fast. An excellent discussion of the concept of normality and its consequences has been given by Bliss [21].

Until recently the concept of normality was either ignored or else strong normality assumptions were imposed. In fact, it was customary to suppose that the arc under consideration was normal on every subinterval and in the sufficiency proofs it was further assumed that an extension of the arc had this property. The effect of these normality assumptions was that the arguments used for simpler problems could be carried over to the problem of Bolza with the obvious modifications. Unfortunately the minimizing arcs of many important problems that are of the Bolza type are necessarily abnormal on every subinterval. For example, this is true for the problem of Mayer. Thus the earlier theories for the problem of Bolza were not applicable to these special cases. A further objection to normality assumptions is that it is difficult in a particular case to verify these assumptions. It would be desirable to proceed without the use of hypotheses of normality and much has been done in this direction. The sufficiency theorems that are now known involve no assumptions of normality. Moreover McShane [26] has shown that a minimizing arc necessarily satisfies (with multipliers $\left.l_{0} \geqq 0, l_{\gamma}(x)\right)$ : (I) the Euler-Lagrange equations and the transversality condition; (II) the condition of Weierstrass; (III) the condition of Clebsch, even if the arc is abnormal. Graves [5] had shown earlier that these results were true for arcs that are normal relative to the end conditions (7) but are not normal on every subinterval as was previously supposed. He also gave rather complicated analogues of the Weierstrass condition for abnormal arcs. As yet it appears to be necessary to assume that the arc under consideration is normal relative to the end conditions in order to obtain an adequate discussion of the fourth necessary condition involving the non-negativeness of the second variation. However, McShane [31] has announced recently that the fourth necessary condition holds when the maximum rank attainable for the matrix (14) is $2 n-1$. Moreover he states that by means of an example it can be shown that the fourth necessary condition in its present form does not hold if this determinant is always of rank at most $2 n-2$.

4. Conditions for a minimum. Consider now a particular arc $E$ without corners that minimizes the function $I$ subject to the conditions (6) and (7). If $E$ is normal, it has associated [24] with it a unique set of multipliers $l_{\gamma}(x)$ with which it satisfies the Euler-Lagrange equations 


$$
(d / d x) F_{y_{i}^{\prime}}=F_{y_{i}}, \quad \phi_{\gamma}=0,
$$

and the transversality condition

$$
d g+\left[\left(F-y_{i}^{\prime} F_{y_{i}^{\prime}}^{\prime}\right) d x+F_{y_{i}^{\prime}} d y_{i}\right]_{1}^{2}+\int_{x_{1}}^{x_{2}} F_{a_{h}} d a_{h} d x=0 .
$$

Here $F=f+l_{\gamma}(x) \phi_{\gamma}$ and equation (16) is an identity in $d a_{h}$ when the differentials $d x_{1}, d y_{i 1}, d x_{2}, d y_{i 2}$ are expressed in terms of $d a_{h}$ by means of equations (7). This is known as the first necessary condition (I) for $E$. Moreover $E$ must satisfy the condition (II) of Weierstrass, that is, at each element $\left(a, x, y, y^{\prime}, l\right)$ belonging to $E$ the inequality

$$
E\left(a, x, y, y^{\prime}, l, Y^{\prime}\right) \geqq 0
$$

must hold for every admissible solution $\left(Y^{\prime}\right)$ of the equations $\phi_{\gamma}\left(a, x, y, Y^{\prime}\right)=0$, where

$$
\begin{aligned}
E= & F\left(a, x, y, Y^{\prime}, l\right)-F\left(a, x, y, y^{\prime}, l\right) \\
& -\left(Y_{i}^{\prime}-y_{i}^{\prime}\right) F_{y_{i}^{\prime}}\left(a, x, y, y^{\prime}, l\right) .
\end{aligned}
$$

From this result one concludes that the further inequality

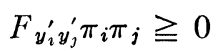

must hold on $E$ for every solution $(\pi)$ of equations $\phi_{\gamma y_{i}^{\prime}} \pi_{i}=0$. This condition is known as the condition (III) of Clebsch. As a fourth necessary condition (IV) the second variation

$$
I_{2}(\alpha, \eta)=b_{h k} \alpha_{h} \alpha_{k}+\int_{x_{1}}^{x_{2}} 2 \omega\left(\alpha, x, \eta, \eta^{\prime}\right) d x
$$

of $I$ along $E$ must be non-negative for every solution $\alpha_{h}, \eta_{i}$ of equations (12) and (13). The last condition can be replaced by conditions involving broken extremals for the second variation.

A solution

$$
a_{h}, \quad y_{i}(x), \quad l_{\gamma}(x), \quad x_{1} \leqq x \leqq x_{2},
$$

of equations (15) is called an extremal. It is said to be nonsingular if the determinant

$$
\left|\begin{array}{cc}
F_{y_{i}^{\prime} y_{j}^{\prime}} & \phi_{\gamma y_{i}^{\prime}} \\
\phi_{\beta y_{j}^{\prime}} & 0
\end{array}\right|
$$

is different from zero at each point on it. Every normal minimizing arc $E$ determines a unique extremal, also designated by $E$, satisfying the necessary conditions described above. When these conditions are 
suitably strengthened they will insure a minimum. For a proper strong relative minimum it is sufficient that $E$ belongs to a nonsingular extremal satisfying the transversality condition (16); the strengthened conditions: $\left(\mathrm{II}_{N}\right)$ of Weierstrass which requires the inequality (17) to hold for all solutions $\left(a, x, y, y^{\prime}, l\right)$ of $\phi_{\gamma}=0$ in a neighborhood $N$ of those on $E$, and (IV') which requires the second variation (20) to be positive for all non-null solutions $\alpha_{h}, \eta_{i}(x)$ of equations (12) and (13). If the Weierstrass condition $\left(\mathrm{II}_{N}\right)$ is replaced by the Clebsch condition (III) described above, a set of sufficient conditions for a weak relative minimum is obtained. In either case the condition $\left(\mathrm{IV}^{\prime}\right)$ can be replaced by conditions involving broken extremals for $I_{2}(\alpha, \eta)$. A detailed account of these and other conditions can be found in the papers of Bliss [13], Hestenes [10, 16], Morse [11] and Reid [12].

5. A consequence of the sufficiency conditions. The sufficiency conditions just described hold even if the arc $E$ is not normal. However, it was pointed out by Professor Bliss that these conditions are essentially a set of sufficiency conditions for a normal problem obtained by enlarging the class of comparison arcs. Thus as far as sufficiency conditions are concerned there is no loss of generality in assuming that the problem is normal. The sufficiency conditions described above also have a further startling consequence. It is clear that our problem will be unaltered if the integral $I$ to be minimized is replaced by an integral of the form

$$
J=g(t)+\int_{x_{1}}^{x_{2}} F\left(a, x, y, y^{\prime}\right) d x,
$$

where $F$ is of the form

$$
F=f+m_{\gamma}\left(a, x, y, y^{\prime}\right) \phi_{\gamma} .
$$

If an $\operatorname{arc} E$ satisfies the sufficiency conditions described above, the multipliers $m_{\gamma}$ can be chosen so that $E$ affords at least a proper weak relative minimum to $J$ in the class of all neighboring arcs satisfying the end conditions ( 7 ) but not necessarily the differential equations (6). Thus in a sense the differential equations $\phi_{\gamma}=0$ can be discarded. The essential step in the proof of this result was supplied by Professor Reid [25], who showed that by taking $m_{\gamma}$ of the form $c \phi_{\gamma}$, where $c$

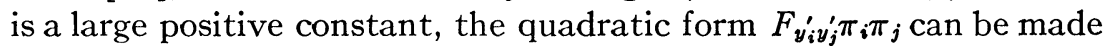
positive definite along $E$. It remains to show that these multipliers $m_{\gamma}$ can be further modified so that the second variation of $J$ is positive definite for all non-null admissible variations $\alpha_{h}, \eta_{i}$ satisfying the end 
conditions (13) irrespective as to whether or not they satisfy the differential equations (12). That this can be done is an immediate consequence of the sufficiency proofs for the problem of Bolza given by Reid [25] and Hestenes [24] as we shall see in a moment.

As one would expect, a similar result holds in the finite case. Consider the problem of minimizing a function $f(x)=f\left(x_{1}, \cdots, x_{n}\right)$ on a surface $\phi_{\gamma}(x)=0(\gamma=1, \cdots, m<n)$. For a proper relative minimum at $(x)=(a)$ it is sufficient that there exist a function $F=f+l_{\gamma} \phi_{\gamma}$ such that at $(x)=(a)$ one has $F_{z_{i}}=0, \phi_{\gamma}=0$ and $F_{x_{i} x_{j}} h_{i} h_{j}>0$ for every solution $(h) \neq(0)$ of the equations $\phi_{\gamma x_{i}} h_{i}=0$. For a sufficiently large constant $c$ the function $G=F+c \phi_{\gamma} \phi_{\gamma}$ will have $G_{x_{i}}=0$ and $G_{x_{i} x_{j}} h_{i} h_{j}$ positive definite at $(x)=(a)$. The point $(x)=(a)$ will accordingly afford a proper minimum to $G(x)$ relative to all points in a neighborhood of $(x)=(a)$. Clearly $G(x)=f(x)$ when $\phi_{\gamma}=0$.

6. Mayer fields. We come now to the consideration as to how the sufficiency theorems can be established. Consider first the case when $g=0$ and none of the parameters $a_{h}$ is present. Then our problem becomes the problem of Lagrange with fixed end points. The method for this case is classical. It consists of constructing a Mayer field. By a Mayer field $\mathcal{F}$ is meant a region $\mathcal{F}$ in $x y$-space together with a set of slope functions and multipliers $p_{i}(x, y), l_{\gamma}(x, y)$ on $\mathcal{F}$ such that $\phi_{\gamma}(x, y, p)=0$ and the Hilbert integral

$$
I^{*}(C)=\int_{C} F(x, y, p, l) d x+\left(d y_{i}-p_{i} d x\right) F_{y_{i}^{\prime}}(x, y, p, l)
$$

is independent of the path in $\mathcal{F}$, where $F=f+l_{\gamma} \phi_{\gamma}$. For any arc $C$ in $\mathcal{F}$ on which $\phi_{\gamma}=0$ one has the relation

$$
I(C)=I^{*}(C)+\int_{C} E\left(x, y, p, l, y^{\prime}\right) d x,
$$

the last integrand being the Weierstrass $E$-function (18). For an arc $E$ in $\mathcal{F}$ on which $y_{i}^{\prime}=p_{i}$, the relation $I^{*}(E)=I(E)$ holds. Thus if an arc $C$ (satisfying $\phi_{\gamma}=0$ ) in $\mathcal{F}$ joins the end points of $E$, one has $I(C) \geqq I^{*}(C)=I^{*}(E)=I(E)$, provided the $E$-function is non-negative along $C$. Consequently $E$ is a minimizing arc when the Weierstrass condition $\left(\mathrm{II}_{N}\right)$ holds.

Returning now to the problem at hand let us recall the sufficiency proof given by Bliss [4] in 1932. This proof combines results that had been established previously by Mayer and Hahn. It consists of three steps: Given an arc $E_{0}$ satisfying the criteria for a minimum it is shown first that there is a neighborhood $\mathcal{F}_{1}$ of $E_{0}$ such that every 
extremal $E$ in $\mathcal{F}_{1}$ affords a minimum to $I(C)$ in the class of arcs $C$ in $\mathcal{F}_{1}$ joining its end points and having $\phi_{\gamma}=0$. Next it is proved that $I\left(E_{0}\right)$ is a minimum on the class of extremals in $\mathcal{F}_{1}$ satisfying the end conditions (7). Finally it is shown that the end points of an arc $C$ in a smaller neighborhood $\mathcal{F}$ of $E_{0}$ can be joined by an extremal $E$ in $\mathcal{F}_{1}$. Hence if $C$ satisfies equations (6) and (7) one has $I(C) \geqq I(E) \geqq I\left(E_{0}\right)$, as desired. A similar method applied to broken extremals was used by Morse [3] in 1931.

The method just described had to be abandoned because the end points of an arc $C$ cannot in general be joined by an extremal $E$ unless restrictive normality conditions are satisfied. For example, the method is not applicable to the problem of Mayer. In order to remedy this situation Bliss and Hestenes [7, 8] devised a method that is applicable to the problem of Mayer, but it too was based on undesirable normality assumptions. A new method accordingly had to be devised. The new method consists essentially of enlarging the concept of Mayer fields. Curiously enough it can be considered to be a generalization of the method used by Bliss, although to the casual observer there may appear to be no connection between the two methods.

Consider now a region $\mathcal{F}$ in $a x y$-space and a set of slope functions and multipliers $p_{i}(a, x, y), l_{\gamma}(a, x, y)$ on $\mathcal{F}$. Set

$$
\begin{gathered}
J(C)=g(a)+\int_{x_{1}}^{x_{2}} F\left(a, x, y, y^{\prime}\right) d x, \\
J^{*}(C)=g(a)+\int_{x_{1}}^{x_{2}} F^{*}\left(a, x, y, y^{\prime}\right) d x,
\end{gathered}
$$

where $F=f+l_{\gamma} \phi_{\gamma}$ and

$$
F^{*}=F(a, x, y, p)+\left(y_{i}^{\prime}-p_{i}\right) F_{y_{i}^{\prime}}(a, x, y, p) .
$$

One then has the formula

$$
J(C)=J^{*}(C)+\int_{C} E\left(a, x, y, y^{\prime}\right) d x
$$

analogous to (23), in which the last integrand is the Weierstrass $E$-function $E=F-F^{*}$. For a solution $C$ of the equations $y_{i}^{\prime}=p_{i}$ the relation $J(C)=J^{*}(C)$ holds. If $\phi_{\gamma}=0$ on $C$ then $J(C)=I(C)$, where $I(C)$ is the function to be minimized. Clearly we can suppose that $I(C) \equiv J(C)$ as far as the problem of Bolza is concerned.

The region $\mathcal{F}$ and the slope functions and multipliers $p_{i}(a, x, y)$, $l_{\gamma}(a, x, y)$ will be said [24] to define a Mayer field $\mathcal{F}$ if the Hilbert 
integral $J^{*}(C)$ is independent of the path in $\mathcal{F}$ in the sense that $J^{*}(C)=J^{*}\left(C^{\prime}\right)$ for any two $\operatorname{arcs} C, C^{\prime}$ in $\mathcal{F}$ having the same end values $\left[a, x_{1}, y\left(x_{1}\right), x_{2}, y\left(x_{2}\right)\right]$. A solution $a_{h}, y_{i}(x)$ of the equations $y_{i}^{\prime}=p_{i}$ defines an extremal $E$ of $J(C)$ and is called an extremal of the field $\mathcal{F}$. The sufficiency proof consists of showing that if an admissible arc $E_{0}$ satisfies the criteria for a minimum, it is an extremal of a Mayer field having the following two properties: The Weierstrass $E$-function $F-F^{*}$ is non-negative for every solution $\left(a, x, y, y^{\prime}\right)$ of $\phi_{\gamma}=0$ having $(a, x, y)$ in $\mathcal{F}$. The arc $E_{0}$ minimizes $J^{*}(C)$ in the class of arcs $C$ satisfying the end conditions (7). Thus if an arc $C$ in $\mathcal{F}$ satisfies equations (6) and (7) one has, by virtue of formula (24), the desired relations $J(C) \geqq J^{*}(C) \geqq J^{*}\left(E_{0}\right)=J\left(E_{0}\right)$. In fact if our problem has been modified so that the quadratic form $F_{y_{i}^{\prime} y_{j}^{\prime}} \pi_{i} \pi_{j}$ is positive definite along $E_{0}$, as Professor Reid has done, then the arc $E_{0}$ not only minimizes $J(C)$ in the class of arcs $C$ in $\mathcal{F}$ satisfying conditions (6) and (7), but affords at least a weak relative minimum in the class of all arcs in $\mathcal{F}$ satisfying the end conditions (7). This establishes the remarks made in $\$ 5$.

I remarked a moment ago that the method just described can be considered to be a generalization of the method used earlier by Bliss [4]. Bliss imposed sufficient normality conditions so that the end points of every arc $C$ in $\mathcal{F}$ can be joined by an extremal $E$. Under these assumptions the field can be chosen [19] so that the relation $J^{*}(C)=J(E)$ holds, provided $C$ satisfies the end conditions (7). Thus the inequality $J^{*}(C) \geqq J^{*}\left(E_{0}\right)$ becomes the criterion that $E_{0}$ minimize $J(E)$ in the class of extremal arcs satisfying the end conditions of our problem.

In order to carry out the method just described it must be known under what conditions the arc $E_{0}$ affords a minimum to $J^{*}(C)$ subject to the end conditions (7). In the theory of Mayer fields we are led therefore to the consideration of an auxiliary minimum problem. In this new problem the parameters $a_{h}$ will in general appear in the integrand of $J^{*}(C)$ even if the original function $I(C)$ does not have this property. Thus by introducing the $a$ 's in the original integral $I$ one can apply at once the theory of the first and second variation to our new problem. Nothing new is obtained from the first variation of $J^{*}(C)$. The second variation $J_{2}^{*}(\alpha, \eta)$ of $J^{*}(C)$ along $E_{0}$ is the Hilbert integral for a Mayer field for the second variation $I_{2}(\alpha, \eta)$ along $E_{0}$. The slope functions and multipliers $\pi_{i}(\alpha, x, \eta), \lambda_{\gamma}(\alpha, x, \eta)$ of this field are the variations along $E_{0}$ of the slope functions and multipliers $p_{i}$, $l_{\gamma}$ of the original field. In this accessory field the second variation $J_{2}(\alpha, \eta)$ of $J(C)$ along $E_{0}$ plays the role of $J(C)$ and is connected to $J_{2}^{*}(\alpha, \eta)$ by the formula 


$$
J_{2}(\alpha, \eta)=J_{2}^{*}(\alpha, \eta)+\int_{x_{1}}^{x_{2}} F_{y_{1}^{\prime} y_{j}^{\prime}}\left(\eta_{i}^{\prime}-\pi_{i}\right)\left(\eta_{j}^{\prime}-\pi_{j}\right) d x
$$

corresponding to formula (26). This formula in a somewhat modified but equivalent form is known as the Clebsch transformation of the second variation. Thus the theory of Mayer fields has a counterpart in the theory of the second variation. If a Mayer field exists for the second variation, then $E_{0}$ can always be imbedded in a Mayer field whose slope functions and multipliers have as their variations along $E_{0}$ the slope functions and multipliers of the given Mayer field for the second variation. The existence of Mayer fields therefore has been reduced to the question of existence of Mayer fields for the second variation. A nonsingular extremal satisfying the transversality conditions and on which the second variation is positive is always an extremal of a Mayer field $\mathcal{F}$ having the properties described above.

7. Expansion proofs. The question naturally arises as to whether or not Mayer fields are essential in the sufficiency proofs for the problem of Bolza. At present I am familiar with only one sufficiency proof for a strong relative minimum that is not based directly on the theory of Mayer fields. This proof is an expansion proof given by Reid [18, $20,25]$ and is a modification and extension of one given by Levi for free problems. Although the expansion proof does not make direct use of the theory of Mayer fields, there is a close relationship between the two methods. In order to emphasize this relationship I shall interpret for you the methods used in the expansion proofs in such a way that one can see more clearly wherein it differs from the more classical method of constructing Mayer fields.

Consider now an extremal $E_{0}$,

$$
a_{h 0}, \quad y_{i 0}(x), \quad l_{\gamma 0}(x), \quad x_{1} \leqq x \leqq x_{2},
$$

satisfying the criteria for a strong relative minimum. We can suppose, with Reid, that the integrand $f$ has been modified so that the quadratic form $F_{y_{i}^{\prime} y_{k}^{\prime}}^{\prime} \pi_{i} \pi_{k}$ is positive definite along $E_{0}$. Then there is a constant $b>0$ such that at each element $\left(a, x, y, y^{\prime}, l\right)$ in a neighborhood $N$ of those on $E_{0}$ the inequality

$$
E\left(a, x, y, y^{\prime}, l, Y^{\prime}\right) \geqq b\left\|Y^{\prime}-y^{\prime}\right\|^{2} /\left(1+\left\|Y^{\prime}-y^{\prime}\right\|\right)
$$

holds whenever $\phi_{\gamma}\left(a, x, y, Y^{\prime}\right)=0$, where $\left\|y^{\prime}\right\|=\left(y_{i}^{\prime} y_{i}^{\prime}\right)^{1 / 2}$. This formula also holds whenever $\left(a, x, y, Y^{\prime}, l\right)$ is in $N$, if $N$ is sufficiently small. Let $\pi_{i}(\alpha, x, \eta), \lambda_{\gamma}(\alpha, x, \eta)$ be the slope functions and multipliers for a Mayer field for the second variation of $I$ whose Hilbert integral $J_{2}^{*}(\alpha, \eta)$ is positive for every admissible variation $(\alpha, \eta) \neq(0, \eta)$ satis- 
fying the end conditions (13). Let $\mathcal{F}$ be a neighborhood of $E_{0}$ in $a x y$ space and select slope functions and multipliers $p_{i}(a, x, y), l_{\gamma}(a, x, y)$ on $\mathcal{F}$ such that $p_{i}=y_{i 0}{ }^{\prime}, l_{\gamma}=l_{\gamma 0}$ along $E_{0}$ and $\pi_{i}, \lambda_{\gamma}$ are the variations of $p_{i}, l_{\gamma}$ along $E_{0}$. As in the theory of Mayer fields construct the functions $J(C), J^{*}(C)$ by means of formulas (24), (25). The integral $J^{*}$ is now no longer independent of the path but its second variation along $E_{0}$ is the Hilbert integral $J_{2}^{*}(\alpha, \eta)$ of the Mayer field for the second variation. The formulas (26) and (27) hold as before. Consider an admissible arc $C$ in $\mathcal{F}$ satisfying the conditions (6) and (7). By the use of Taylor's formula for $J^{*}(C)$, the properties of $J_{2}^{*}(\alpha, \eta)$ and the inequality (29) one finds after considerable manipulation that the relation

$$
J^{*}(C)-J^{*}\left(E_{0}\right)+\int_{C} E d x \geqq 0
$$

holds, provided $\mathcal{F}$ is taken sufficiently small. By the use of equations (26) and $J^{*}\left(E_{0}\right)=J\left(E_{0}\right)$ this inequality becomes $J(C)-J\left(E_{0}\right) \geqq 0$, as desired.

The expansion proof given by Reid was presented in quite a different manner. However, from the above interpretation it is seen that it has much in common with the theory of Mayer fields. In particular it is seen that the basic property for $J^{*}(C)$ is that its second variation along $E_{0}$ is the Hilbert integral for the second variation of $I(C)$ and is positive for all variations $(\alpha, \eta) \neq(0, \eta)$ satisfying the variational end conditions (13). As far as the problem of Bolza is concerned it appears that the theory of Mayer fields is the simpler. However the expansion method has the advantage that it appears to be applicable to problems for which Mayer fields fail to exist.

8. Further results. The inequality (29) has many applications. For example, it can be used to prove the following analogue of Osgood's theorem: Let $E_{0}$ be a nonsingular extremal satisfying the criteria for a strong relative minimum described above. There exists a neighborhood $\mathcal{F}$ of $E_{0}$ in $a x y$-space such that corresponding to every neighborhood $\mathcal{F}$ of $E_{0}$ interior to $\mathcal{F}$, there is a constant $\epsilon>0$ such that the inequality $I(C) \geqq I\left(E_{0}\right)+\epsilon$ holds for every arc $C$ in $\mathcal{F}$, not in $\mathcal{F}^{\prime}$, which satisfies equations (6) and (7). This result was established by Reid [25] by expansion methods. It can also be proved by the use of Mayer fields with the help of the relations (26) and (29).

There is a second important result that is a consequence of the inequality (29). It is a generalization by Reid [25] of a theorem of Lindeberg which states that if $E_{0}$ is a nonsingular extremal (28) satis- 
fying the condition $\left(\mathrm{II}_{N}\right)$ of Weierstrass, and $\rho$ is a positive constant, there is a neighborhood $\mathcal{F}$ of $E_{0}$ such that $J(C)-J\left(E_{0}\right)>\rho / 2$ holds for every admissible arc $C$ in $\mathcal{F}$ for which one has

$$
\int_{C} E\left(a, x, y, y_{0}^{\prime}, y^{\prime}\right) d x>\rho,
$$

where $y_{0}^{\prime}(x)$ are the slope functions of $E_{0}$. By the use of this result and one other, that I shall not describe here, Reid was able to obtain an elegant proof of the sufficiency theorems for the isoperimetric problem of Bolza. The sufficiency conditions for this problem are analogous to those for the problem of Bolza. Only two changes need to be made. First, the new function $F$ appearing in the various conditions is now of the form $F=f+\lambda_{\gamma}(x) \phi_{\gamma}+\lambda_{m+\rho} f_{\rho}$, where $\lambda_{m+\rho}(\rho=1, \cdots, p)$ are constants. Similarly $g$ is to be replaced by $g+\lambda_{m+\rho} g_{\rho}$. The second difference is that the variations $\alpha_{h}, \eta_{i}$ must satisfy besides the conditions (12) and (13), the additional conditions

$$
\begin{aligned}
J_{\rho 1}(\alpha, \eta)= & g_{\rho a_{h}} \alpha_{h}+\left[f_{\rho} x_{s a_{h}} \alpha_{h}\right]_{s=1}^{s=2} \\
& +\int_{x_{1}}^{x_{2}}\left\{f_{\rho a_{h}} \alpha_{h}+f_{\rho y_{i}} \eta_{i}+f_{\rho y_{i}^{\prime} \eta_{i}^{\prime}}\right\} d x=0
\end{aligned}
$$

where the functions $J_{\rho 1}(\alpha, \eta)$ are the variations along $E_{0}$ of the functions $J_{\rho}$ appearing in the side conditions (9). A second sufficiency proof for the isoperimetric problem of Bolza has been given by Hestenes $[24]$.

9. Indices of extremals. Returning now to the problem of Bolza let us consider a normal nonsingular extremal $E_{0}$ satisfying the end conditions (7), the transversality condition (16) and the condition (III) of Clebsch. If the second variation of $I$ is positive along $E_{0}$, then $E_{0}$ affords at least a proper weak relative minimum to $I(C)$. The question naturally arises as to whether or not $E_{0}$ has certain minimizing properties even if the second variation is not positive along $E_{0}$. The answer is in the affirmative. The extremal $E_{0}$ is a minimizing arc for a normal isoperimetric problem of Bolza obtained by adjoining conditions of the form (9). The least number $k$ of side conditions of this type which will determine an isoperimetric problem of Bolza for which $I\left(E_{0}\right)$ is a minimum will be called the index of the extremal $E_{0}$ (cf. [15]). The condition that the problem remain normal is an essential one since otherwise one could adjoin the trivial condition $I(C)=I\left(E_{0}\right)$ and the concept of index would not be a useful one. Clearly the index of $E_{0}$ is zero if and only if $E_{0}$ is a minimizing arc for the original problem of 
Bolza. Except in certain degenerate cases the adjoined side conditions can be taken to be of the special form

$$
\begin{aligned}
I_{1}\left(\alpha_{\rho}, \eta_{\rho} ; C\right)= & g_{a_{h}} \alpha_{h_{\rho}}+\left[F x_{s a_{h}} \alpha_{h_{\rho}}\right]_{1}^{2} \\
& +\int_{x_{1}}^{x_{2}}\left\{F_{a_{h}} \alpha_{h}+F_{g_{i}} \eta_{i}+F_{y_{i}^{\prime}} \eta_{i}^{\prime}\right\} d x=0
\end{aligned}
$$

where $\alpha_{h_{\rho}}, \eta_{i \rho}(\rho=1, \cdots, k)$ form a set of $k$ variations satisfying the equations (12) and (13) and the elements $\left(a, x, y, y^{\prime}\right)$ are those belonging to $C$. The expression $I_{1}\left(\alpha, \eta ; E_{0}\right)$ is the first variation of $I$ along $E_{0}$ and is zero for all variations satisfying the conditions (12), (13).

In order to determine the index $k$ of $E_{0}$ it is sufficient to select the fewest number of conditions (9) or (32) preserving normality which are such that the second variation $I_{2}(\alpha, \eta)$ is positive along $E_{0}$ for all non-null variations $\alpha_{h}, \eta_{i}$ satisfying the conditions (12), (13) and (31). For then the criteria for a weak relative minimum will be satisfied. It will be seen in the following sections that the second variation in general can be made positive if the variations $\alpha_{h}, \eta_{i}$ are required to satisfy, besides the conditions (12) and (13), a set of $k$ additional conditions of the form

$$
b_{h j} \alpha_{h \rho} \alpha_{j}+\int_{x_{1}}^{x_{2}}\left\{\omega_{\alpha_{h}} \alpha_{h \rho}+\omega_{\eta_{i}} \eta_{i \rho}+\omega_{\eta_{i}}^{\prime} \eta_{i \rho}^{\prime}\right\} d x=0, \quad \rho=1, \cdots, k,
$$

where $\alpha_{h_{\rho}}, \eta_{i \rho}$ are $k$ solutions of equations (12) and (13). The fewest number of conditions (33) that are effective in this manner is called the index of $I_{2}(\alpha, \eta)$ and in general is equal to the index of $E_{0}$. This follows because the equations (33) are the variations along $E_{0}$ of the equations (32).

10. Indices of the second variation. In order to discuss the index of the second variation it is convenient to change our notation somewhat and return to the formulation used by Bliss. We consider the class $\mathcal{D}$ of all continuous arcs

$$
\eta: \quad \eta_{i}(x), \quad x_{1} \leqq x \leqq x_{2} ; i=1, \cdots, n,
$$

having piecewise continuous derivatives that satisfy differential equations of the form

$$
\Phi_{\gamma}\left(\eta, \eta^{\prime}\right)=M_{\gamma i} \eta_{i}+N_{\gamma i} \eta_{i}^{\prime}=0, \quad \gamma=1, \cdots, m<n,
$$

whose coefficients are continuous functions of $x$ on $x_{1} x_{2}$. On this class there is defined a quadratic function 


$$
J(\eta)=2 q(\eta)+\int_{x_{1}}^{x_{2}} 2 \omega\left(\eta, \eta^{\prime}\right) d x
$$

where $2 q$ is a quadratic form in the end values $\eta_{i}\left(x_{1}\right), \eta_{i}\left(x_{2}\right)$ of $\eta$ and

$$
2 \omega=P_{i j}(x) \eta_{i} \eta_{j}+2 Q_{i j}(x) \eta_{i} \eta_{j}^{\prime}+R_{i j}(x) \eta_{i}^{\prime} \eta_{j}^{\prime} .
$$

It is assumed that the functions $P_{i j}=P_{j i}, Q_{i j}, R_{i j}=R_{j i}$ are continuous on $x_{1} x_{2}$ and that the determinant

$$
\left|\begin{array}{ll}
R_{i j} & N_{\beta i} \\
N_{\gamma j} & 0
\end{array}\right|
$$

is different from zero. The bilinear form $J(\xi, \eta)$ associated with $J(\eta)$ is given by the formula

$$
J(\xi, \eta)=q_{\eta_{i 1}} \xi_{i}\left(x_{1}\right)+q_{\eta_{i} \xi_{i}} \xi_{i}\left(x_{2}\right)+\int_{x_{1}}^{x_{2}}\left\{\omega_{\eta_{i}} \xi_{i}+\omega_{\eta_{i}}^{\prime} \xi_{i}^{\prime}\right\} d x
$$

and is identical with the first variation of $J(\eta)$. We have $J(\xi, \eta)=J(\eta, \xi)$ and $J(\eta, \eta)=J(\eta)$. Finally let $\varepsilon$ be the class of all variations $\eta$ in $\mathcal{D}$ satisfying end conditions of the form

$$
\Psi_{\mu}(\eta)=a_{\mu i} \eta_{i}\left(x_{1}\right)+b_{\mu i} \eta_{i}\left(x_{2}\right)=0, \quad \mu=1, \cdots, p \leqq 2 n .
$$

We are interested in the properties of $J(\eta)$ on $\mathcal{E}$. Other classes $\varepsilon$ are also of interest. The problem of the second variation $I_{2}(\alpha, \eta)$ of $I$ described in the preceding sections is readily reduced to one of this type.

Many of the results that follow are equally applicable to a quadratic function on an arbitrary linear space. However as we shall be interested in the interpretations of these results in the problem of Bolza, I shall restrict myself to the problem here described. The results here given are a combination of the results obtained by Birkhoff and Hestenes [15] and Hazard [29].

Two $\operatorname{arcs} \xi$ and $\eta$ will be said to be $J$-orthogonal if the relation $J(\xi, \eta)=0$ holds. An arc $\xi$ will be said to be $J$-orthogonal to a subclass $\mathcal{E}^{*}$ of $\mathcal{D}$ if it is $J$-orthogonal to every arc in $\mathcal{E}^{*} . J$-orthogonal classes are defined similarly. By the $J$-complement of a subclass $\mathcal{E}^{*}$ of $\mathcal{E}$ in $\varepsilon$ will be meant the totality of $\operatorname{arcs}$ in $\eta$ in $\varepsilon$ that are $J$-orthogonal to $\varepsilon^{*}$. Similarly by the $J$-complement of an arc $\xi$ in $\varepsilon$ will be meant the set of all arcs in $\varepsilon$ that are $J$-orthogonal to $\xi$. The condition that $\xi$ be an extremal is that it be $J$-orthogonal to the class of all arcs $\eta$ in $\mathscr{D}$ vanishing at $x_{1}$ and $x_{2}$. This is equivalent to the definition of extremals given previously if one identifies extremals which differ only in their 
multipliers. An extremal is seen to satisfy the transversality condition used in the previous sections if and only if it is $J$-orthogonal to $\varepsilon$.

In the last section the index of the second variation was defined in terms of an isoperimetric problem of Bolza. There is a second definition of index that is a more convenient one and which is more easily understood. The index of $J(\eta)$ on $\varepsilon$ will be called the $J$-index of $\varepsilon$. By the $J$-index of a linear subclass $\varepsilon^{*}$ of $\mathcal{D}$ will be meant the dimension of a maximal linear subclass of $\mathcal{E}^{*}$ on which $J(\eta)$ is negative definite.

This number is easily seen to be well defined. In view of the nonvanishing of the determinant (36) the $J$-index of $\varepsilon$ will be finite if and only if the Clebsch condition (III) holds. We shall be interested only in the case in which $J$-indices are finite. We shall accordingly assume that the Clebsch condition holds.

In order to see that the index here defined is identical with the one given earlier, let $\xi_{1}, \cdots, \xi_{k}$ be a minimal basis for a maximal linear manifold $\varepsilon$ - in $\varepsilon$ on which $J(\eta)$ is negative definite. Then an arc $\eta$ in $\mathcal{E}$ is $J$-orthogonal to $\mathcal{E}$ - if and only if the conditions $J\left(\xi_{\alpha}, \eta\right)=0$ $(\alpha=1, \cdots, k)$ hold. It follows from our choice of $\mathcal{E}$ - that $J(\eta) \geqq 0$ for every arc in $\varepsilon$ satisfying these isoperimetric conditions. Moreover no fewer conditions have this property. The two concepts of index are therefore identical. There is a further defining property of the $J$-index of a class $\varepsilon^{*}$. The $J$-index of $\varepsilon^{*}$ is equal to the dimension of a maximal linear subclass of $\varepsilon^{*}$ on which $J(\eta) \leqq 0$ and which contains no arc $\eta \neq 0$ that is $J$-orthogonal to $\mathcal{E}^{*}$. This is a very useful criterion for the determination of indices.

One of the basic problems is the determination of the relations between the $J$-index of $\varepsilon$ and that of a linear subclass $\varepsilon^{*}$ of $\varepsilon$. This problem can be completely solved if $\mathcal{E}^{*}$ is related to $\mathcal{E}$ in a special way. For want of better terminology, these subclasses will be called special subclasses of $\varepsilon$. Let $\varepsilon^{*}$ be a linear subclass of $\varepsilon$ and denote by $E_{0}^{*}$ the set of all arcs in $\varepsilon^{*}$ that are $J$-orthogonal to $\varepsilon^{*}$. The class $\mathcal{E}^{*}$ will be called a special subclass of $\mathcal{E}$ if every arc $\eta$ in $\mathcal{E}$ that is $J$-orthogonal to $\varepsilon_{0}^{*}$ is a sum $\eta_{1}+\eta_{2}$ of an arc $\eta_{1}$ in $\varepsilon^{*}$ and an arc $\eta_{2}$ that is $J$-orthogonal to $\varepsilon^{*}$. For example the subclass $\varepsilon^{0}$ of all $\operatorname{arcs}$ in $\varepsilon$ vanishing at $x_{1}$ and $x_{2}$ is a special subclass of $\varepsilon$. Here the condition that $\varepsilon^{0}$ be a special subclass of $\varepsilon$ is equivalent to the condition that the end points of every arc $\eta$ in $\varepsilon$ that is $J$-orthogonal to the extremals that vanish at $x_{1}$ and $x_{2}$ can be joined by an extremal. Every linear subclass of $\mathcal{E}$ containing $\mathcal{E}^{0}$ is a special subclass of $\mathcal{E}$. In fact any linear subclass of $\varepsilon$ of finite dimension or which is the $J$-complement of such a class is a special linear subclass of $\varepsilon$. 
The principal result upon which the determination of indices and upon which many comparison theorems are based is the following one: Let $\varepsilon^{*}$ be a special linear subclass of $\varepsilon$ and as before denote by $\varepsilon_{0}^{*}$ the class of all arcs in $\varepsilon^{*}$ that are $J$-orthogonal to $\mathcal{E}^{*}$. Let $m$ be the dimension of a maximal linear subclass of $\varepsilon_{0}^{*}$ whose non-null arcs are not $J$-orthogonal to $\varepsilon$. If the $J$-indices of $\varepsilon^{*}$ and its $J$-complement in $\varepsilon$ be denoted by the symbols $k^{*}$ and $k^{\prime}$, respectively, then the $J$-index $k$ of $\varepsilon$ is given by the formula $k=k^{*}+k^{\prime}+m$.

As a first example of the application of this result let us consider the problem of determining the $J$-index of the class $\varepsilon^{0}$ of all $\operatorname{arcs} \eta$ in $\mathcal{D}$ vanishing at $x_{1}$ and $x_{2}$. For convenience of description it will be assumed that $\eta \equiv 0$ is the only extremal that vanishes identically on any subinterval of $x_{1} x_{2}$. This is a normality condition and need not be assumed if one modifies the definition of conjugate points given below. A point $x_{3}$ on $x_{1}<x<x_{2}$ will be said to be conjugate to $x_{1}$ if there is an extremal $\eta \neq 0$ that vanishes at $x_{1}$ and $x_{3}$. The number $h$ of linearly independent extremals in a maximal set that vanish at $x_{1}$ and $x_{3}$ will be called the order of $x_{3}$ as a conjugate point of $x_{1}$. Let $\xi_{1}, \cdots, \xi_{m}$ be a maximal set of linearly independent arcs in $\varepsilon^{0}$ that are identically zero on a subinterval $x_{3} x_{2}$ of $x_{1} x_{2}$ and are identical with an extremal on $x_{1} x_{3}$. The number of these arcs is equal to the sum of the orders of the conjugate points of $x_{1}$ between $x_{1}$ and $x_{2}$. These arcs form the basis of a special subclass $\varepsilon^{*}$ of $\varepsilon^{0}$ whose index is zero. No $\operatorname{arc} \xi \neq 0$ in $\varepsilon^{*}$ is $J$-orthogonal to $\varepsilon^{0}$. Moreover the index of its $J$-complement in $\varepsilon^{0}$ is also zero. The $J$-index of $\mathcal{E}^{0}$ is accordingly equal to the dimension $m$ of $\varepsilon^{*}$ and hence is equal to the sum of the orders of the conjugate points of $x_{1}$ between $x_{1}$ and $x_{2}$.

Returning now to the original class $\varepsilon$ we observe that its $J$-index is the sum of three quantities. The first of these quantities is the index of the class $\varepsilon^{0}$ just described, that is, the sum of the orders of the conjugate points of $x_{1}$ between $x_{1}$ and $x_{2}$. The second quantity is the $J$-index of the class of extremals in $\varepsilon$, that is, the $J$-index of the $J$-complement of $\mathcal{E}^{0}$ in $\mathcal{E}$. The third quantity is the dimension of a maximal linear class of extremals vanishing at $x_{1}$ and $x_{2}$ which contains no extremal $\xi \neq 0$ that satisfies the transversality conditions for $\varepsilon$.

As a third example consider the class $\varepsilon^{*}$ of all arcs in $\varepsilon$ forming the $J$-complement of the class of all arcs vanishing at a set of points $t_{0}=x_{1}<t_{1}<\cdots<t_{r}<t_{r+1}=x_{2}$. These points can be chosen so close together that the index of the $J$-complement of $\varepsilon^{*}$ is zero and such that every arc in $\mathcal{E}^{*}$ that is $J$-orthogonal to $\mathcal{E}^{*}$ is also $J$-orthogonal to $\varepsilon$. The $J$-index of $\mathcal{E}$ is therefore identical with that of $\mathcal{E}^{*}$. 
Moreover the class $\varepsilon^{*}$ is composed of the totality of broken extremals in $\varepsilon$ with corners at the points $t_{1}, \cdots, t_{r}$. This result identifies our index with the one introduced by Morse.

Examples of this type can be multiplied in many ways so as to obtain comparison and oscillation theorems which relate the $J$-indices of various subclasses of $\mathcal{E}$. Other oscillation and comparison theorems are also easily obtained. For example, if a second quadratic function $J^{*}(\eta)$ satisfies the condition $J^{*}(\eta) \geqq J(\eta)$ on $\varepsilon$, the $J^{*}$-index of $\varepsilon$ cannot exceed its $J$-index.

Boundary value problems arise in the calculus of variations when one wishes to obtain relationships between $J(\eta)$ and a second quadratic functional $J^{*}(\eta)$. The function $J^{*}(\eta)$ is usually taken to be of the form

$$
J^{*}(\eta)=2 q^{*}(\eta)+\int_{x_{1}}^{x_{2}} K_{i j}(x) \eta_{i} \eta_{j} d x .
$$

We shall assume that $J^{*}(\eta)$ is positive on $\mathcal{D}$ although this assumption

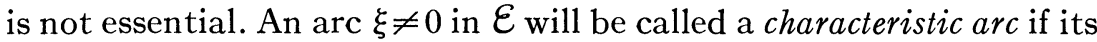
$J^{*}$-complement in $\varepsilon$ is identical with its $J$-complement in $\varepsilon$. If $\xi$ is a characteristic arc, there is a value $\sigma$ such that $J(\xi, \eta)=\sigma J^{*}(\xi, \eta)$ on $\varepsilon$. In fact $\sigma=J(\xi) / J^{*}(\xi)$, and $\xi$ is an extremal for $J-\sigma J^{*}$. The number of linearly independent characteristic arcs in a maximal set determining the same characteristic value $\sigma$ will be called the order of $\sigma$. It can be shown that the $J$-index of $\varepsilon$ is equal to the sum of the orders of the negative characteristic values. Thus it is seen that the theory of indices here presented is closely related to boundary value problems.

\section{REFERENCES}

The following list of references contains only these directly referred to in the present paper. Further references will be found in these papers, particularly in the papers $10,13,14,17,20,22,24$ and 25.

1. O. Bolza, Über den anormal Fall beim Lagrangeschen und Mayerschen Problem mit gemischten Bedingungen und variablen Endpunkten, Mathematische Annalen, vol. 74 (1913), pp. 430-446.

2. G. A. Bliss, The problem of Mayer with variable end points, Transactions of this Society, vol. 19 (1918), pp. 305-314.

3. M. Morse, Sufficient conditions in the problem of Lagrange with variable end conditions, American Journal of Mathematics, vol. 53 (1931), pp. 517-546.

4. G. A. Bliss, The problem of Bolza in the calculus of variations, Annals of Mathematics, (2), vol. 33 (1932), pp. 261-274.

5. L. M. Graves, On the Weierstrass condition for the problem of Bolza in the calculus of variations, Annals of Mathematics, (2), vol. 33 (1932), pp. 747-752.

6. C. Carathéodory, Über die Einteilung der Lagrangeschen Variationsprobleme nach Klassen, Commentarii Mathematici Helvetici, vol. 5 (1933), pp. 1-10.

7. G. A. Bliss and M. R. Hestenes, Sufficient conditions for the problem of Mayer in the calculus of variations, Transactions of this Society, vol. 35 (1933), pp. 305-326. 
8. M. R. Hestenes, Sufficient conditions for the general problem of Mayer with variable end points, Transactions of this Society, vol. 35 (1933), pp. 479-490.

9. W. R. Reid, Analogues of the Jacobi condition for the problem of Mayer in the calculus of variations, Annals of Mathematics, (2), vol. 35 (1934), pp. 836-848.

10. M. R. Hestenes, Sufficient conditions for the problem of Bolza in the calculus of variations, Transactions of this Society, vol. 36 (1934), pp. 793-818.

11. M. Morse, Sufficient conditions in the problem of Lagrange without assumptions of normalcy, Transactions of this Society, vol. 37 (1935), pp. 147-160.

12. W. T. Reid, The theory of the second variation for the problem of Bolza, American Journal of Mathematics, vol. 57 (1935), pp. 573-586.

13. G. A. Bliss, The problem of Bolza in the calculus of variations, mimeographed notes of lectures delivered at the University of Chicago, 1935.

14. - The evolution of problems in the calculus of variations, American Mathematical Monthly, vol. 43 (1936), pp. 598-609.

15. G. D. Birkhoff and M. R. Hestenes, Natural isoperimetric conditions in the calculus of variations, Duke Mathematical Journal vol. 1 (1935), pp. 198-286.

16. M. R. Hestenes, On sufficient conditions in the problems of Lagrange and Bolza, Annals of Mathematics, (2), vol. 37 (1936), pp. 543-551.

17. W. T. Reid, Boundary value problems in the calculus of variations, this Bulletin, vol. 43 (1937), pp. 633-666.

18. - Sufficient conditions by expansion methods for the problem of Bolza in the calculus of variations, Annals of Mathematics, (2), vol. 38 (1937), pp. 662-678.

19. M. R. Hestenes, $A$ direct sufficiency proof for the problem of Bolza in the calculus of variations, Transactions of this Society, vol. 42 (1937), pp. 141-154.

20. W. T. Reid, A direct expansion proof of sufficient conditions for the non-parametric problem of Bolza, Transactions of this Society, vol. 42 (1937), pp. 183-190.

21. G. A. Bliss, Normality and abnormality in the calculus of variations, Transactions of this Society, vol. 43 (1938), pp. 365-376.

22. E. J. McShane, Recent developments in the calculus of variations, American Mathematical Society Semicentennial Publications, vol. 2; Semicentennial Addresses.

23. C. P. Brady, The minimum of a function of integrals in the calculus of variations, dissertation, The University of Chicago, 1938.

24. M. R. Hestenes, Generalized problem of Bolza in the calculus of variations, Duke Mathematical Journal vol. 5 (1939), pp. 309-324.

25. W. T. Reid, Isoperimetric problems of Bolza in non-parametric form, Duke Mathematical Journal, vol. 5 (1939), pp. 675-691.

26. E. J. McShane, On multipliers for Lagrange problems, American Journal of Mathematics, vol. 61 (1939), pp. 809-819.

27. — An estimate of the Weierstrass E-function, Annals of Mathematics, (2), vol. 41 (1940), pp. 314-320.

28. - A remark concerning sufficiency theorems for the problem of Bolza, this Bulletin, vol. 46 (1940), pp. 698-701.

29. K. Hazard, Index theorems for the problem of Bolza in the calculus of variations, dissertation, The University of Chicago, 1940.

30. E. J. McShane, Necessary conditions in generalized curve problems of the calculus of variations; existence theorems for Bolza problems in the calculus of variations, Duke Mathematical Journal, vol. 7 (1940), pp. 1-61.

31. - On the second variation in certain anormal problems of the calculus of variations, this Bulletin, abstract 46-5-209.

University of Chicago 